\section{Electronic Publishing Expands}

Electronic publishing continues to expand with the introduction of several new services for physicists. It is difficult to cover all the developments as things evolve very quickly, so here are a few items to illustrate what is happening.

\section{On-line bibliographic databases}

The on-line interrogation of databases via networks is well established and in the case of scientific literature, several organizations operate abstracting services to make available headers (titles, affiliations, abstracts, catalogue data, subject coding, etc.). The number of journals covered continues to grow and suppliers are rationalising and consolidating. For example, the American Institute of Physics (AIP) announced last October that INIST in France will make headers available on-line via Questel, Dialog and ESA

Publishers have themselves started offering on-line search capabilities directly to journal subscribers. For instance, members of the Society of Photo-Optical Instrumentation Engineers (SPIE) can scan the headers of all SPIE journals via the main ftp (file transfer protocol) file archive at mom.spie.org. To reduce costs for European users, there will be a "mirror" site at the University of Salzburg. Meanwhile, North-Holland started up Nuclear Physics Electronic in January to provide on-line index-searching and browsing of Nuclear Physics A \& B headers. Access is via anonymous $\mathrm{ftp}$, email or the World-Wide Web. One needs to subscribe to Nucl. Phys. (or be a member of an institute that does) to read abstracts of papers (scanning indexes is free-of-charge).

A less sophisticated service is offered by the publishers of The Astrophysical Journal Part 2. Headers of accepted Letters can be read via modem or networks (Internet and SPAN) by dial-up to +1 (617) 4957031 with username "apjlett" (at the terminal server prompt Xyplex type "c cfax", where $x=3,4$, 5,7 , or 8 , and then "apjlett" for a username again). Internet users can telnet to cfax. harvard.edu ( $x$ as before).

\section{Alerting/current awareness}

Electronic journal alerting services are only just getting started. Nuclear Physics Electronic (NPE) offers a partial service whereby a list of papers to be published in Nucl. Phys.

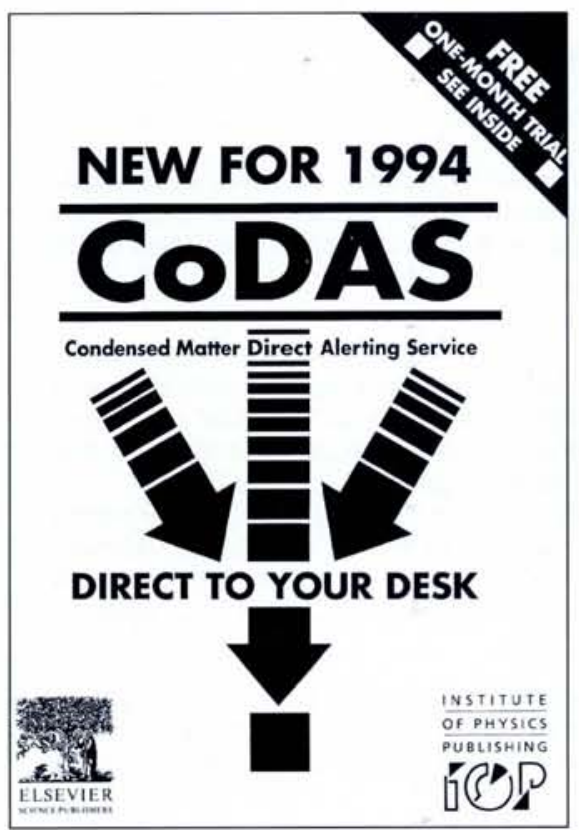

is sent by email free-of charge to anyone interested (one must send an email message to npa-serv@npe.nuclphys.nl with "get readme" in the subject line or contact NPE via d.greaves@ npe.nucphys.nl). Similarly, subscribers to Electronics Letters published by the Institution of Electrical Engineers (IEE) can automatically receive each week by email notification of newly published Letters in particular fields (contact inspec @ dm.rs.ch for details). A similar current awareness service called Advanced Abstracts involves subscribers to AIP'S PINET network receiving abstracts of AIP and its member society journals three months before publication.

A full alerting service is now available to Direct Alerting Service) set up by the Institute of Physics Publishing and Elsevier. Headers of some 55 journals are delivered every week to a computer via the academic networks some 2-3 weeks before publication in the printed form. Software for searching and filtering is also supplied via the networks.

\section{Document delivery}

Abstracts are often available electronically in conjunction with services that offer the searching, browsing or downloading of headers. Going beyond this represents a major step and electronic journals where the entire contents of publications are made available electronically to subscribers on searchable databases remain rare. Searchable header databases such as CODAS generally rely on non-electronic delivery with maybe ordering by email. Impetus for change comes from the are popping up everywhere. North-Holland has made a first step with Nuclear Physics Electronic which allows subscribers to download on-line all Nucl. Phys, abstracts as well as the texts of articles which were submitted electronically and published within the last six months. Meanwhile, the IEE has made Electronics Letters Online - a daily updated version of Electronics Letters - available at the Online Computer Library Center via Internet or dial-up communications using the OCLC's graphical user interface. Some of the features offered are remarkably sophisticated (full-text searching, typeset quality for local printing, hypertext links with links to abstracts in the INSPEC abstracts database, linking of comments to the original Letters.

Major libraries provide electronic document delivery and some publishers have turned to commercial operators. For example, starting last year, the Institute of Electronic and Electrical Engineers (IEEE) teamed up with Dynamic Information Corp. to deliver by email any of the many documents stored by the IEEE. Not to be forgotten are the few general journals offering electronic delivery. For example, the AIP will download upon request to subscribers to its Physics Information Network (PINET), articles published in Physics Today and Computers in Physics.

\section{Moderated bulletin boards}

The Journal of Materials Science has moved some way towards a refereed electronic journal by creating a bulletin board for subscribers which is "moderated" by a group subscribers to CODAS (Condensed Matter preprint and "grey literature" servers which at the University of Michigan. The server contains accepted articles with the referees' comments attached.

\section{Newsletters}

Specialised newsletters are beginning to complement printed editions with electronic versions. For example, an electronic version of the ICO Newsletter published by the International Commission on Optics is sent automatically to subscribers, but without figures. To subscribe one simply sends a one-line message "subscribe iconewsxxyour_NamexxYour_First_Name" to listserve @ frmop11. Others organizations are placing the entire contents of newsletters in the public domain using computer archives which can be easily accessed by anonymous $\mathrm{ftp}$. This is the case for TUG News published by the American Mathematical Society to encourage the use of the $T_{E} X$ typesetting language. The American Physical Society has announced that as from January 1995 members will be able to choose between receiving either the printed verion of its revamped, tabloid-style APS News or an electronic version without halftone figures at their email address. The IOPP will reportedly offer this spring by email Physics World Electronic - a complement to Physics World - to subscribers and Institute of Physics members on a trial basis.

\section{Author services}

Starting this January, the American Physical Society, the publisher of Phys. Rev., Phys. Rev. Lett. and Rev. Modern Phys., has made available on public-domain directories the various items needed by referees and authors. The sub-directories accessible via $\mathrm{ftp}$ to aps.org (the Internet address is 149.28.1.2) include:

- Style, notation and authors' guides and referee response forms (/pub/jnrls directory);

- A classification scheme as an ASCII file (file pacs_94.asc in the /pub/pacs directory). $T_{E} X$ and Postscript files will soon be released and the documents are also available in ASCII and Wordperfect via ftp to pinet.aip.org (192.58.150.10) in the /pub/pacs directory. - Style files for the APS-developed LAT $X$ macro package for compuscripts (/pub/revtex directory).

This last facility is being offered by an increasing number of publishers. For example, $T_{E} X$ and/or LAT $E$ X macro packages comprising public-domain sets of style files can be ordered by sending an email message as follows:

- Z. Physik and Applied Physics (SpringerVerlag): svserv@vax.ntp.springer.de; Nuclear Physics (North-Holland): nhpnuclear @ elsevier.nl; Journal de Physique (Editions de Physique): edphys @ frmop22.bitnet; IEEE Transactions on Electron Devices (IEEE): help@ep.ieee.org.

Finally, style files are starting to become available on servers. For example:

- Journal of the Optical Society of America: a REVT $_{E} X 3.0$ package is obtainable by ftp from niord.shsu.edu or pinet.org and by sending an email to fileserve@ @shsu.edu with the command "sendme revtex" in the body.

- Physics Letters (Elsevier): the ESP-LATEX package is available by anonymous ftp from the Comprehensive $T_{E} X$ Archive Network (CTAN) directories at three hosts (e.g., Host name: ftp.uni-stuttgart.de; Directory:/pub/tex macros/latex/contrib/elsevier).

\section{P.G. Boswell}

Secretary, EPS Publications Committee 